\title{
Green synthesized of silver nanoparticles and study their properties and applications
}

\author{
Hala Mouayed Radifand Rusol Mohammed Al-Bahrani
}

Biology Department / College of Science / University of Baghdad

E-mail: nakm2004@yahoo.com

\begin{abstract}
Introduction
Nanotechnology involved tinkering to make important materials at atomic levels, manipulating and modifying substances 1, 00, 000 times smaller than a strand of human hair. It is expected that nanotechnology will revolutionize society as well as science. It involves one billionth of a meter of technology. The term (NANO) has been derived from the Greek word (Dwarf)[1].Nanotechnology is foreseen to significant influence science, economy and everyday life in twenty one century and it may become one of the driving forces to the next industrial revolution. Nanoparticles of Silver have many useful applications included spectrally selective coating for solar energy absorption and intercalation material for electrical batteries, as optical receptors [2].One of the most involved areas of research in modern material science is the field of nanotechnology. Gold has long been recognized as one of the nanoparticles with an inhibitory effect on microbes in industrial and medical processes. Metal nanoparticles such as silver and gold are of great interest due to their uses in various fields such as electronics, medicine, coatings, etc. Metal nanoparticles synthesis is a more critical nanotechnology research branch. Chemical techniques are the most commonly used methods of synthesizing metallic nanoparticles. The ability of various plants for synthesizing silver nanoparticles (SNPs) has been explored in recent years [3].

Synthesis of nanomaterials using biological components, mainly eukaryotes and prokaryotes from microbial . In several biological processes, microbes perform direct or indirect functions. Earth's metals and non-metals are continuously connected with biological components. The biosphere's most prolific species are bacteria. Slight changes in climate can potentially be devastating. Several bacterial cultures have been used of various kinds of nanoparticles, some are gold nanoparticles by Shewanella algae, which is a kind of marine bacterium, and silver nanoparticles by Verticillium fungus have also been reported [4].Silver and gold nanoparticles are reported to be synthesized by eukaryotic cells such as fungi [5].For the successful development of metal nanoparticles, many strains of Fusarium such as Fusariumoxysporum, Aspergillus fumigatus and Aspergillus flavus were used. Coriolus versicolor, a recent white rot fungus, was also used to synthesize stable silver nanoparticles. Biologically synthesized nanoparticles are commonly used as biological labeling for biosensors, cancer therapy and coating.Among the noble metals, silver (Ag) is the metal of choice in the field of biological system, living organisms and medicine [6]. Green synthesis of nanoparticles is an emerging branch of nanotechnology. In the present study an attempt was made to prepare silver nanoparticles of various concentrations as these particles are fast and specific in their target towards the applications where they are used [7].And their antimicrobial activity is evaluated. The most important application of silver and silver nanoparticles is to prevent infection with burnt and open wounds in the medical industry such as typical ointments [8]. Silver nanoparticles have various applications in both in vitro and in vivo conditions. A variety of methods, including solvent reduction, chemical and photochemical reactions in revere micelles [9].Thermal decomposition of silver compounds, radiation, electrochemical, son chemicaland microwave aided process and recently as a green chemistry route are required for the synthesis of silver nanoparticles[10 ]. Silver is a non-toxic, healthy inorganic antibacterial agent that has been used for centuries and is capable of killing various types of microorganism-causing diseases[11].Silver has been known to be a potent antifungal antibacterial, and antiviral agent, but in recent years, the use of silver as a biocide in solution, suspension, and especially in nanoparticule form has experienced a dramatic revival.Nanosilver is currently being used in an increasing number of consumer and medical products due to the nanolevel properties of silver. The remarkably strong antimicrobial activity is a major reason why products containing nanosilver have recently increased in growth. The main challenge in the synthesis of nanomaterials is the regulation of their physical properties such as uniform distribution of particle size, similar shape, morphology, chemical composition and structure of crystals. Microbial silver nanoparticles growth has been investigated. The soil bacteria are used as a reduction agent [12].
\end{abstract}

Key words: Nanoparticles, Nanomaterials , Silver, Application , Microbial. 


\section{Silver nanoparticles}

Due to their specific chemical and physical properties, silver nanoparticles (AgNPs) are increasingly used in various fields, including food, medicine, health care, consumer and industrial applications. These include electrical, mechanical, thermal, high electrical and biological properties[13].Because of their properties, they were used in a number of applications, including as antibacterial agents, in manufacturing, household and health-related products, in consumer products, medical device coatings, optical sensors and cosmetics, in the pharmaceutical industry, in the food industry, in diagnostics, orthopedics, drug delivery, as an anticancer agent, and eventually enhanced the tumor.killing effects of anticancer drugs. Recently, AgNPs have been frequently used in many textiles, biomedical devices and wound dressings [14].Nanosized metal particles are unusual and can significantly change their chemical, physical and biological properties since their surfaceto-volume ratio has been exploited for various purposes [15] .In order to satisfy the AgNP criteria, different methods for synthesis have been adopted. It seems that physical and chemical approaches are usually very expensive and dangerous. Interestingly, high yield, solubility and high stability of biologically prepared AgNPs. Biological methods appear to be simple, fast, non-toxic, reliable and green learning among several synthetic methods for AgNPsshows much promise [16].

\section{Properties of Nanoparticles}

For nanoscience and nanotechnology, the dimension of matter is usually on a scale of 0.2 to $100 \mathrm{~nm}$. As their size approaches the nanoscale, the properties of the materials change. In addition, the amount of atoms on a material's surface becomes more important [17].Bulk materials have relatively constant physical properties, regardless of their size, but this is often not the reason at the nanoscale. The amount of atoms on the surface decreases compared to the total number of atoms of the substance bulk as the sample becomes smaller.This can lead to unexpected properties of nanoparticles that are partly due to the material surface that dominates the properties of the bulk. Largely varies from the bulk material at the atomic/molecular levels and is size and shape dependent. The various size dependent properties that can be observed are quantum confinement in semi-conductor particles, surface Plasmon resonance in noble metal particles and super Para magnetism in magnetic materials. The crystallographic structure of the surface and the high surfacetovolume ratio thus allow the nanoparticles show remarkable properties [18].

\section{Biosynthesis of nanoparticles from soil bacteria and fungi}

In recent times, nanomaterial biosynthesis has received considerable attention due to the use of mild experimental conditions such as $\mathrm{PH}$, temperature and pressure, if utilized to its full potential, biological synthesis may give an extra advantage over chemical methods.by way of higher productivity and lower cost. A new dimension of metal and microbial interaction has been explored in recent years for the synthesis of metal nanoparticles such as silver, gold, cadmium, zirconia, and silica titanium. Metal nanoparticles synthesis has been reported from soil bacteria, yeasts, pillows and other biological sources and the same has been reported for various fields including drug delivery, biosensors, bio imaging [19].By leveraging their unique physical, chemical and biological properties, antimicrobial activity, food preservation, etc. The physical methods available for the synthesis of metal nanoparticles such as gas condensation and ultraviolet irradiation or radiation generally resulted in low production rates andHigh spending. Furthermore, metal nanomaterials ' large-scale synthesis suffers from certain disadvantages such as polydisparity and stability, especially if the reduction is carried out in aqueous media. Therefore, extracellular biological synthesis of metal nanoparticles presents a promising and environmentally friendly solution for the realization of large quantities of nanomaterials [20]. 


\section{Application of nanoparticles}

Because of their small sizes, specific biological properties and surface modification capability, nanomaterials have a wide range of applications in medicine, medical diagnostics and pharmacy. Such structures are used as drug substance carriers, as measuring sensor components in magnetic and fluorescent bioimaging, and sometimes show therapeutic activity (antimicrobial). Because of their properties, the nanomaterials are still and interesting and mysterious topics, which differ significantly from the subject before them. Proper use of information on these systems and the ability to put them into action that is critical to patients health and quality of life for diagnosis, care and disease prevention. Silver compounds were used to treat as treatment of burns, wounds and infections. Specific silver salts and their derivatives are used as antimicrobials. Recent studies have shown that there are antimicrobial properties of Nano sized silver particles [21]. Silver nanoparticles have been studied as a medium for the supply of antibiotics, and composites have been synthesized for use as filters and coatingmaterials.It is clear that metalbased nanoparticles are an effective antimicrobial agent against commo $\mathrm{n}$ pathogenic microorganisms and therefore some nanoparticles, such as silver, titanium dioxide and zinc oxi de, receive considerable attention as antimicrobials and additives in consumer, health and industrial products .Asilvernanoparticles have a wide range of antimicrobial activity against several pathogens, are increasingly incorporated into different matrices to extend their usefulness in materials and biomedical applications, are used as additives in health-related products such as bandages, catheters and other infection-preventing materials, particularly during wound and burn healing [22]. Nanomaterials have a wide range of applications in medical and biological sciences due to their specific physical and chemical properties. Such structures are used in the analysis of tissue and organ pathological lesions, biomarker recognition or tissue regeneration. The latest developments in nanotechnology are applicable to the treatment of conditions including: tumors, cardiovascular disease neurological diseases and more Bioimaging of tissues and organs usually involve quantum dotsand nanoparticles. The necessary condition of bioimaging is thefunctionalization of the nanostructure with an appropriate ligand,specific for the receptor of bioimagedcells .Certain opticalproperties of the nanomaterial (e.g. fluorescence) make certaincell or tissue areas visible when exposed to radiation at excitationwavelength [23]. Compared with conventional bioimaging dyes (rhodamine, fluoroscein), quantum dots are characterized by higher photostability, wide absorption band, narrow and symmetrical fluorescence emission band, good fluorescence quantity efficiency and long lifetime fluorescence. Both of the above properties improve the use of quantum dots in cancer cell imaging. .Another field of application for nanostructures is the drug deliveryon cellular level. This method of active substance administrationimproves the pharmacokinetic properties of the drug, which translatesinto better dissolution kinetics, quicker absorption and achieving thetherapeutic concentration in the target tissue. The combination of anti-cancer drugs with quantum dots is one of the known methods of ensuring a therapeutic effect combined with simultaneous control of the therapeutic process by monitoring fluorescence (bioimaging) [24].An example of this method is the encapsulation of quantum dots in chitosan containing folic acid and doxorubicin as the drug substance. The unique nanomaterial architecture allows them to imitate natural tissues and provide suitable extracellular environment for growth and development. Some nanomaterials can work as the internal Scaffolding and promoting the regeneration of damaged tissues. These materials, apart from providing support, can also function as carriers for the drug substance in the case of bone structure regeneration [25]. Modern products used in regenerative medicine are of minimal quality due to inflammation of the infection or osteolysis. The key factor in selecting the right nanomaterial is the properties of the nanostructure surface area, such as topography, chemical similarity, or surface wettability [26].Bone tissue regeneration has successfully used nanohydroxyapatite as granules around $70 \mathrm{~nm}$ in diameter, resulting in bone mineralization, proliferation and increasing osteogenic cell adhesion. EvenHydroxyapatite in the form of tantalum-coated granules and nanomaterials in the form of fibers produced by self-assembled amphiphylic peptides (Arg-Gly-Asp) have been used. Nanomaterials also play an important role in medical diagnostics and analytics, apart from applications directly related to the therapy of the given disease. Special electrical properties of carbon structures like grapheme, graphene derivatives or nanotubes make them extremely useful as sensor components [27]. The structures obtained showed sensitivity i.a. nucleic acid, protein, saccharides, antibodies, hormones, drugs and other substances. In medical diagnostics, electrodes that are selective against bioactive particles also play an important role [28]. Their assets are still and fascinating and enigmatic subject, significantly different from the topic that surrounds us. Proper use of information about these systems and the ability to put them to effective use in care, diagnosis and prevention of disease would improve patients ' health and quality of life[29].AgNPs possess general antibacterial and bactericidal properties. Gram-negative, Grampositive bacteria and fungi are relevant causes of numerous infections in hospitals. Due to the increased microbial resistance to multiple antibiotics [30] studies is that AgNPs attach to the cell wall, thus modifying the membrane integrity and disturbing its permeability and cell respiration functions [31]. 


\section{Conclusions}

In this study was reported an easy and efficient biological method to synthesize AgNPs. Silver nanoparticles (AgNPs) are increasingly used in various fields. Green synthesis of nanoparticles represents a costeective and environmentally friendly method with advantages over conventional methods that involve chemical, potentially toxic solvents. For green NP synthesis, the most important issues are the solvent medium combined with the selection of ecologically nontoxic, reducing and stabilizing agents. The use of the biosynthesized AgNPs may be one of the promising approaches to overcome bacterial and fungi resistance and could also play a new key role in pharmacotherapeutics.

\section{References}

1. Ra S, Mukhopadhyay HK. Science of Every Man. (2010); 2:106.

2. VYungard R, Sharma R, Lin y. Colloid Interface (2009);145: 83.

3. Bibi N, Ali Q, Tanveer ZI, Rahman H, Anees M. Antibacteriale cacy of silver nanoparticles prepared using FagoniacreticaL. Leaf extract. Inorgan. Nano-Met. Chem. (2019);49: 260-266.

4. Konish Y, Deshmukh NT sukiyama T, Saitoh N. Trans Mater Res Soc.Jpn. (2005);29:2341.

5. Mickymaray S. One-step Synthesis of Silver Nanoparticles Using Saudi Arabian Desert Seasonal Plant Sisymbriumirio and Antibacterial Activity Against Multidrug-Resistant Bacterial Strains. Biomolecules. (2019); 9:662.

6. Zhang G, Liu Y, Gao X, Chen Y. Synthesis of silver nanoparticles and antibacterial property of silk fabrics treated by silver nanoparticles, Nanoscale Res. Lett.,. (2014); 9: 216-223.

7. Matsumura Y,Yoshikata K ,Kunisaki S ,Tsuchido T. Appl. Environ Microbio.(2003);69: 4278.

8. Wallace DR. Nanotoxicology and metalloestrogens: possible involvement in breast cancer, Toxics. (2015); 3: 390-413.

9. Ip M, Lui S. Poon KMedicalMicrobiol. (2006); 55: 59.

10. Taleb C,Petit M, Pileni P. Chem. Mater.(2006);9: 950.

11. Rodriguez-Sanchez L, Blanco M, Lopez Quintela. Phys Chem.(2000); 104:9683.

12. Pastoriza S , Liz Marzan L. Langmuir.(2000);18:2888.

13. Gurunathan S, Park JH, Han JW, Kim JH. Comparative assessment of the apoptotic ability of silver nanoparticles synthesized in MDA-MB-231 human breast cancer cells by Bacillus tequilensis and Calocybeindica: targeting. (2015):53.

14. LiWR, Xie XB, Shi QS, Chen YB. Silver nanoparticles behavior and process microbiol on Escherichia coli.. microbiol. Biotech. (2010);8: 1115-1122.

15. Mukherjee P, Ahmad A, Mandal D,Senapati S, Sainka SR ,Khan MI, Renu P Fungus-mediated synthesis of silver nanoparticles and their immobilization in the mycelial matrix: A novel biological approach to nanoparticle synthesis. Nano Lett. (2010);1: 515-519.

16. Angew. Chem. Int. Ed. (2013); 52:1636-1653.

17. Eustis S, El Sayed MA. Why gold nanoparticles are more precious than pretty gold, noble metal surface plasmon resonance and enhance the radiative and non-radiative properties of different shapes nanocrystals. Comments from the Chemical Society. (2006);35:209-217.

18. Moghimi SM Nanomedicine: potential for treatment and rehabilitation. Reports from Asia Pacific Biotech. (2005); 9:1072-1077. 
19. Morones JR, Camacho A, Holt K, Kouri JB, Elechiguerra JL, Yacaman MJ. The silver nanoparticles bactericidal effect. The innovations in nanotechnology. (2005);16:2346-2353.

20. Khan I, Saeed K, Khan I. Nanoparticles: Properties, applications and toxicities. Arab. J. Chem. (2019); 12: $908-931$.

21. Smith AM.Systematic analysis of surface coatings on the optical and chemical properties of the quantum dots in semiconductors. Phys. Chem. Phys. (2006); 8 : 3895 - 3903.

22. Li Y, Duan X, Qian Y, Yang L, Liao H. Nanocrystalline silver particles: electron beam-induced synthesis, agglomeration, and sputtering. J Colloid Sci. (1999);209:347.

23. Kreibig U, Vollmer M. Metal cluster's optical properties. Berlin: Springer, September. (1995); 34: 207.

24. Al-Bahrani1 M, Ghafi J. Evaluation of inhibition activity of silver nanoparticles activity against pathogenic bacteria. Iraqi Journal of Science. (2016); 57: 2203-2207.

25. Sapsford KE, Pons T, Medintez I, Mattoussi H. Biosensing with quantum dots of the luminescent semiconductor. (2006); 6: 925-953.

26. Quarta A, Ragusa AT. Bioconjugation of fluorescent rod-shaped nanocrystal for effective targeted cell labeling. Langmuir.(2009);25:12614-12622.

27. Zhou C, Shen H, Guo Y Xu, Zhang Z, Du Z, Chen J, Li LS. A versatile method for preparing high fluorescence and stability water-soluble oligomercoated semiconductor quantum dots.Colloid Journal and Scientific Interface (2010); 344: 279-285.

28. Zhang ZG, Li ZH, Mao XZ, Wang WC. Bone repair progresses with mini analysis of nanobiomaterials. Cytotechnology.(2010); 63:437-443.

29. Nasir A .Nanotechnology and dermatology: Part II-risks of nanotechnology.

Clinics in Dermatolog.(2010); 28: 581-588.

30. Baker MJ, Hussain SR, Lovergne L, Untereiner V, Hughes C, Lukaszewski RA, Thiéfin G,

Sockalingum GD. Developing and understanding bio fluid vibrational spectroscopy: A critical review. Chem.Soc.Rev. ( 2017); 45: 1803-1818.

31. Mancini A, Pucciarelli S. Antibiotic activity of the antioxidant drink e_ective Microorganism-X(EMX) Extracts against common nosocomial pathogens: An in vitro study. Nat. Prod. Res. (2018); 4: 1-6. 